\title{
Société médicale du canton du Jura
}

Remo Osterwalder

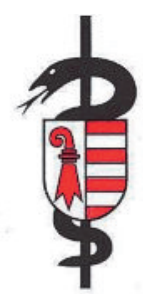

SOCIÉTÉ MÉDICALE DU CANTON DU JURA

Correspondance:

Dr Remo Osterwalder Président de la SMCJU Rue du 24-Septembre 7

CH-2800 Delémont

Tél. 0324234040

Fax 0324234044

\section{Historique}

Sur le plan de sa forme actuelle et de sa structure, notre société est l'une des plus jeunes, car elle remonte à la création du canton du Jura en 1979. A l'origine, nous étions rattachés à nos confrères du Jura bernois qui ont aujourd'hui encore leur propre section au sein de la Société des médecins du canton de Berne. Nous avons de bons contacts avec nos confrères du Jura sud, relations que nous entretenons par l'organisation d'une Assemblée générale commune à la fin de chaque année. A l'heure actuelle notre société compte 152 membres, dont une centaine travaille en pratique privée ou en cabinet de groupe. Parmi eux, 50 détiennent un titre de spécialiste en médecine générale ou en médecine interne.

\section{Problèmes}

En tant que canton limitrophe et canton romand le plus au nord de la Suisse, nous sommes confrontés à des problèmes spécifiques touchant surtout la médecine de premier recours. Pour de nombreux Romands, nous sommes loin des centres urbains tels que Lausanne et Genève. Nous collaborons surtout étroitement avec le canton de Neuchâtel (p. ex. commission d'éthique, pandémie) et aussi avec Bâle, le centre universitaire le plus proche.

Les difficultés de la médecine de premier recours et plus particulièrement la question de l'organisation du service de garde ont imposé l'élévation de la limite d'âge à 65 ans pour les médecins tenus de prendre part à ce service et un projet de rattachement, voire de collaboration avec l'hôpital cantonal du Jura est actuellement à l'étude. Cela permettrait de décharger les généralistes et d'accroître l'attrait pour la profession. De plus, une population d'environ 70000 habitants (env. $0,9 \%$ de la population suisse) et une superficie de $838 \mathrm{~km}^{2}$ (2\% du territoire suisse) accentuent la difficulté sur le plan géographique. La relation médecinpatient est cependant d'autant plus intéressante et solide car, consciente des distances importantes, la population attribue un rôle important à une prise en charge locale de qualité.

\section{Perspectives d'avenir}

Les régions périphériques font à nouveau les frais d'une politique de santé devenue incontrôlable. Comment rendre la médecine de premier recours attrayante pour les jeunes médecins, lorsque mois après mois, les mauvaises nouvelles concernant la diminution et la détérioration de la profession redoublent d'intensité. La frustration est immense et les efforts pour attirer de jeunes médecins suisses bien formés sont vains.

Nous n'attendons pas que Berne nous propose la panacée, mais une solution fédéraliste axée sur les difficultés sans remarques déplacées ni sanctions globales. Nous connaissons bien nos difficultés et nos besoins, nous voulons et pouvons trouver des solutions. La distribution directe de médicaments par le médecin est p. ex. réglée sur le plan régional et individuel et fonctionne parfaitement bien. Cela se remarque également dans la structure des coûts de la santé dans le Jura. La collaboration entre le canton et les médecins fonctionne à merveille et se veut complémentaire. Les difficultés des grands centres ne doivent pas être résolues sur le dos des régions périphériques. Dès que la politique de santé tiendra à nouveau compte des spécificités régionales, nous pourrons nous recentrer sur notre activité principale au service du patient. Nous avons besoin de jeunes médecins et nous demandons où est la relève. Pourquoi les autorités n'investissentelles pas plus d'argent dans la relève de sorte à garantir une prise en charge médicale de qualité? 